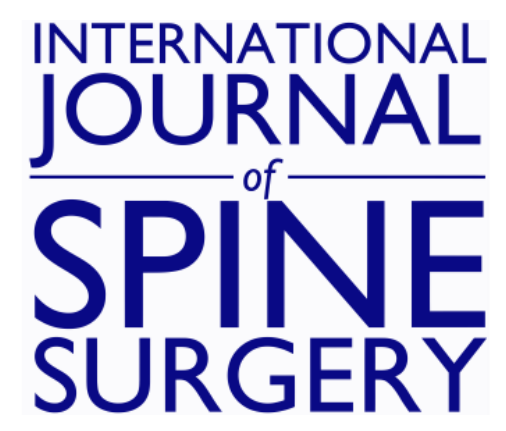

\title{
ISASS Policy 2016 Update - Minimally Invasive Sacroiliac Joint Fusion
}

Morgan P. Lorio

Int J Spine Surg 2016, 10 ()

doi: https://doi.org/10.14444/3026

http://ijssurgery.com/content/10/26

This information is current as of April 26, 2023.

Email Alerts Receive free email-alerts when new articles cite this article. Sign up at:

http://ijssurgery.com/alerts

The International Journal of Shing Surgerh http://ijssurgery.com/ by guest on April 26, 2397 Waterbury Circle, Suite 1,

Aurora, IL 60504, Phone: +1-630-375-1432

(C) 2016 ISASS. All Rights Reserved. 


\section{ISASS Policy 2016 Update - Minimally Invasive Sacroiliac Joint Fusion}

Morgan P. Lorio, MD, FACS

ISASS Coding \& Reimbursement Task Force Chair

\section{Rationale}

The index 2014 ISASS Policy Statement - Minimally Invasive Sacroiliac Joint Fusion was generated out of necessity to provide an ICD9-based background and emphasize tools to ensure correct diagnosis. A timely ICD10-based 2016 Update provides a granular threshold selection with improved level of evidence and a more robust, relevant database.

KEYWORDS: ISASS, POLICY STATEMENT, MINIMALLY INVASIVE SURGERY, SACROILIAC JOINT, FUSION, COVERAGE

\section{Introduction}

The sacroiliac joints (SIJ) are diarthrodial articulations of the sacrum and ilium. The SIJ serves as the biomechanical mediator between the spine and pelvis. The subchondral bone, capsule, and surrounding ligaments of the SIJ are innervated by spinal nerves. ${ }^{1}$

Sacroiliac joint (SIJ) pain is likely responsible for chronic back pain in some patients; furthermore in some studies the prevalence is reported to be $15-30 \% .{ }^{2-6}$ Convergence of the sensory pathway from the hip, the SIJ and the lumbar spine may result in overlap of pain patterns from dysfunction of these structures. As such, proper SIJ pain diagnosis is key to appropriate patient management. Patients with SIJ pain typically report pain in the buttock(s), with possible radiation into the groin or upper legs. Specific physical examination tests that stress the SIJ (e.g., distraction test, compression test, thigh thrust, FABER (Patrick's) test, Gaenslen's maneuver) are typically performed in the physician's office; in combination, these tests are thought to be predictive of SIJ pain. ${ }^{7}$

The spectrum of pain and disability from SIJ dysfunction is wide. Patients may be affected mildly or may have substantial functional impairment (e.g., cannot sit or stand for more than five minutes, cannot perform normal activities of daily living (ADLs), cannot walk up or down stairs, may require a wheelchair). Patients with chronic SIJ dysfunction seeking surgical treatment have marked impairment of quality of life, ${ }^{8}$ similar to that observed in other conditions commonly treated surgically. ${ }^{9}$ Apart from ankylosing spondylitis, in which MRI can show edema consistent with inflammation, imaging of the SIJ typically does not provide valuable diagnostic information. In many cases, imaging can show non-specific findings in the SIJ. ${ }^{10}$ Rather, imaging is used to ensure that the patient does not have alternative diagnoses that could mimic SIJ pain (e.g., hip osteoarthritis, occasionally L5/S1 spine degeneration) (Table 1 ).

The diagnosis of SIJ pain is confirmed by performing a fluoroscopy-guided percutaneous SIJ block with local anesthetic (e.g., lidocaine). An acute reduction in typical pain indicates a positive test, suggesting that the injected joint is a pain generator. A study of patients undergoing blinded injection of saline or local anesthetic showed markedly high responses to the latter, validating the test. ${ }^{11}$ Because other pathologic processes can coexist with SIJ pain, physicians should discuss with patients the degree to which treatment of the SIJ may relieve overall pain and disability without addressing other pain generators.

Occasionally, bilateral SIJ pain can occur. Diagnosis of bilateral SIJ pain should be made on the basis of typical history (bilateral symptoms), physical examination showing positive responses to SIJ-stressing maneuvers bilaterally, and bilateral acute pain relief upon bilateral, fluoroscopy-guided SIJ block.

While a marked response to SIJ block might be pre- 
dicted to reassure the physician that treatment will produce larger responses to anatomic-based treatment, published data suggest little, if any, relationship. In two large prospective clinical trials of SIJ fusion, patients with suspected SIJ pain were included only if intraarticular SIJ block resulted in a $50 \%$ or greater amount of acute pain relief within 60 minutes after the block. The degree of improvement at 6 and 12 months after SIJ fusion was unrelated to the degree of acute pain relief during the block. ${ }^{12}$ In a retrospective analysis of predictors of outcome success after RF ablation of lateral branches of the sacral nerve roots in patients with SIJ pain, no relationship was observed between response to lateral branch block or SIJ anesthesia and response to RF ablation. ${ }^{13}$ Randomized trials of $\mathrm{RF}$ ablation of lateral branches of the sacral nerve roots excluded patients with $<75 \%$ pain reduction after lateral branch block (one block in Cohen et al. ${ }^{14}$ and two blocks in Patel et al. $\left.{ }^{15}\right)$, leaving open the question of whether the selected threshold was appropriate.

Multiple non-surgical treatments for SIJ pain are available, including pain medications (e.g., nonsteroid anti-inflammatory agents), physical therapy, steroid injections into the SIJ, and radiofrequency ablation of the sacral nerves and SIJ fusion. While pain medications may relieve temporarily pain and/or disability, they have not been shown to impact the underlying disease process, and opioid addiction remains an important public health concern. Apart from a single clinical trial in post-partum pelvic pain (probably related to the SIJ), ${ }^{16}$ the effectiveness of physical therapy for chronic SIJ dysfunction has not been demonstrated. Two randomized trials have shown that RF ablation of lateral branches of sacral nerve roots can temporarily reduce SIJ pain. ${ }^{14,15}$ Oneyear follow-up from one $\mathrm{RF}$ ablation randomized trial showed modest pain reduction. ${ }^{17}$ Responses in the non-surgical arms of two prospective randomized trials showed little, if any, improvement at 6 months. ${ }^{18,19}$ Given the absence of published outcomes data supporting long-term pain relief from non-surgical treatment, patients with a diagnosis of SIJ pain who experience pain for a minimum of six months and who do not respond to an adequate course of non-surgical treatment may be considered for SIJ fusion.
Coverage Rationale for Open and Minimally Invasive SIJ Fusion

Open fusion of the SIJ, first reported in the early $1900 \mathrm{~s},{ }^{20}$ can provide pain relief but recovery times are long and complication rates are high, ${ }^{21-25}$ intraoperative times, bleeding and hospital length of stay are more prominent compared to minimally invasive SIJ fusion, ${ }^{26}$ and recovery times are long and may require prolonged postoperative rehabilitation. Therefore, open fusion of the SIJ is best performed on patients who are not candidates for minimally invasive SIJ fusion.

Minimally invasive fusion of the SIJ has been performed with several types of implants, including triangular, porous, titanium coated implants, ${ }^{19,27-33}$ hollow modular screws, ${ }^{34-36}$ titanium cages, ${ }^{37,38}$ and allograft dowels ${ }^{21}$ (Table 2). Minimally invasive fusion aims to permanently stabilize the SIJ but avoid the morbidity of the open procedure.

Two surgical approaches are commonly used for minimally invasive SIJ fusion:

- A lateral transarticular approach, in which devices are placed across the SI joint from lateral to medial. Multiple devices are FDA cleared for this approach for conditions including sacroiliac joint disruptions and degenerative sacroiliitis. However, the vast majority of the published clinical literature for this approach reports use of triangular titanium implants (iFuse Implant System, SI-BONE, Inc.).

- A posterior approach, in which devices are placed into the ligamentous portion of the joint via dissection of the multifidus muscle and removal of ligaments covering the outer posterior surface of the joint. In the posterior approach, a portion of the interosseous SIJ ligament is sometimes removed.

\section{Published Literature}

Published outcomes data for minimally invasive SIJ fusion using a posterior approach are scarce. One cohort reported marginal response to use of cages placed into the SIJ through a posterior approach. ${ }^{38}$ For the lateral approach, 3 retrospective case series (describing two cohorts) using hollow modular anchor (HMA) screws suggest reasonable 2- and 3-year outcomes. ${ }^{35,36}$ HMA screws are not FDA-cleared for 
SIJ fusion.and are not available for use in the U.S. The remaining published literature on SIJ fusion through a lateral approach used triangular titanium implants (iFuse Implant System, SI-BONE, Inc.). This literature includes:

- A US multicenter, randomized clinical trial (INSITE, $\mathrm{n}=148)^{19}$ with an embedded cost-utility analy$\operatorname{sis}^{39}$

- A European multicenter, randomized clinical trial (iMIA, $\mathrm{n}=103$, in press $^{18}$ )

- A US prospective multicenter single-arm clinical trial ( $\mathrm{n}=172)$ with 24-month follow-up ${ }^{31}$

- Several single-center case series ${ }^{26,27,40-44}$

- A multicenter case series ${ }^{32}$

- 3 comparative studies comparing open and iFusebased SI joint fusion ${ }^{26,45,46}$

- An analysis of implant survivorship ${ }^{47}$

- A systematic review and meta-analysis ${ }^{48}$

- A systematic review. ${ }^{49}$ Both systematic reviews focused on laterally-based procedures and products.

The majority of cohorts were triangular titanium implants.

Taken together, these studies provide substantial evidence that minimally invasive SIJ fusion with triangular titanium implants improves pain, function and quality of life. In both randomized trials, pain relief, disability reduction and improvement in quality of life were markedly higher in SIJ fusion subjects compared to non-surgically treated subjects. Specifically, in the SIJ fusion group of the US randomized trial, ${ }^{19}$ mean SIJ pain improved from 82.3 at baseline to 30.4 at the 6-month follow-up (52.0-point improvement, $\mathrm{p}<.0001$ ) and 28.3 at the 12-month follow-up (54.2-point improvement, $\mathrm{p}<.0001)$. Mean changes in the non-surgical group were not clinically significant (mean 12 points). Similarly, in the SIJ fusion group, mean ODI decreased from 57.2 at baseline to 29.9 at month 6 and 28.1 at month 12 (improvements of 27.4 and 29.3 points, respectively). In contrast, mean ODI decreased by only 4.6 points in the nonsurgical group. In the European randomized trial, ${ }^{18}$ Mean pain scores improved in the SIJ fusion group from 77.7 at baseline to 34.4 at 6 months (a 43.3 point improvement $\mathrm{p}<.0001$ ) vs. 73.0 to 67.8 (an improvement of 5.7 points, $\mathrm{p}=.1105$ ) in the non-surgical group. ODI improved by 20 points more in the surgi- cal vs. non-surgical groups 9p<.0001). EQ-5D time trade-off index also improved more in the surgical vs. non-surgical group.

In a multicenter retrospective review of 263 patients undergoing either open or minimally invasive SIJ fusion with triangular titanium implants, minimally invasive SIJ fusion was associated with statistically significant and clinically marked decreases in operating room time (mean 163 minutes for open vs. 70 minutes for minimally invasive), decreased blood loss (mean $288 \mathrm{cc}$ vs. $33 \mathrm{cc}$ ), and decreased length of stay (5.1 vs. 1.3 days) as well as improved relief of pain at 1 (-2.7 points on $0-10$ scale vs. -6.2 points) and 2 -year (-2.0 vs. -5.6 points) follow-up (all differences are statistically significant.). ${ }^{26}$ Finally, two published studies report that favorable outcomes achieved at one year are sustained long term (up to 5 years). ${ }^{30,33}$

The complication rate for minimally invasive SIJ fusion with triangular titanium implants is low. ${ }^{50}$ Revision rates over 4 years $\left(3.5 \%{ }^{47}\right)$ are substantially lower than after lumbar fusion surgery, and revision rates in long-term retrospective $\mathrm{e}^{30,33}$ and prospective studies $^{19,31}$ have confirmed this low rate. Revisions can be required in the immediate postoperative period or after many months. Early revisions may include the need to reposition an implant that is impinging on a sacral nerve or removal of an implant due to infection. Revision rates with other products are unknown. Screw-based devices rely upon different fusion strategies (HA coating, fenestrations within the screws, etc.) with different biomechanics (threaded screws vs. triangular implants that are impacted across the SI joint). Regardless of implant, salvage revision remains challenging.

\section{Bilateral Procedures}

In cases of bilateral SIJ pain, bilateral SIJ fusion may occasionally be indicated and is usually performed serially to minimize the impact on rehabilitation (i.e., patients who undergo simultaneous bilateral fusion procedures may be wheelchair or bedbound for several weeks, possible slowing overall recovery).

Downloaded from http://ijssurgery.com/ by guest on April 26, 2023 


\section{Indications/Limitations of Coverage}

Patients who have all of the following criteria may be eligible for minimally invasive SIJ fusion:

- Significant SIJ pain that impacts quality of life or significantly limits activities of daily living;

- SIJ pain confirmed with at least 3 physical examination maneuvers that stress the SIJ (see list provided above) and reproduce the patient's typical pain.

- Confirmation of the SIJ as a pain generator with $\geq 50 \%$ acute decrease in pain upon fluoroscopically guided diagnostic intra-articular SIJ block using local anesthetic. Prospective trials have shown that patients with SIJ pain responses of 50-75\% respond to MIS SIJ fusion as well as those with $75-100 \%$ acute responses. ${ }^{12}$

- Failure to respond to at least 6 months of nonsurgical treatment consisting of non-steroidal antiinflammatory drugs and physical therapy. Failure to respond means continued pain that interferes with activities of daily living and/or results in functional disability;

- Additional or alternative diagnoses that could be responsible for the patient's ongoing pain or disability have been considered. Physicians should take into account that patients can have multiple pain generators and addressing just one pain generator may not adequately relieve disability or all back pain.

Minimally invasive SIJ fusion is NOT indicated for patients with the following:

- Less than 6 months of SIJ pain and/or functional impairment

- Failure to pursue conservative treatment of the

SIJ (unless contra-indicated);

- Pain not confirmed with a diagnostic SIJ block;

- Presence of other pathology that would substantially prevent the patient from deriving benefit from SIJ fusion

Bilateral SIJ pain is not uncommon. Diagnosis of bilateral SIJ pain must be made on the basis of a history of bilateral pain, bilateral elicitation of pain on physical examination maneuvers that stress each SIJ, and acute bilateral decrease in pain upon fluoroscopically-guided intra-articular SIJ block with local anesthetic. Bilateral SIJ fusion is probably best performed serially as successful treatment of one side may improve pain/disability to a degree acceptable by the patient. SIJ fusion of the contralateral side may be necessary if contralateral SIJ pain continues and disability is significant for the patient. If bilateral fusion is performed at the same operative session, the surgeon must document both medical necessity and why serial fusion is not indicated in the patient.

It is expected that a person would not undergo more than one SIJ fusion per side per lifetime except in the rare case that a revision is needed.

\section{Coding}

The American Medical Association recommends minimally invasive SIJ fusion be coded using CPT code 27279. Revision and/or removal of the SIJ implant would typically be coded using 22899 (unlisted procedure, spine) or 27299 (unlisted procedure, pelvis or hip joint) depending on the type of approach and procedure performed, whether within the global period of the fusion, or not.

\section{Documentation Requirements}

- A complete history and physical documenting the likely existence of SIJ pain;

\begin{tabular}{|l|r|}
\hline $\begin{array}{l}\text { Table 1. ICD-10-CM Diagnosis. } \\
\text { ICD-10-CM } \\
\text { Diagnosis } \\
\text { Code }\end{array}$ & Code Descriptor \\
\hline M46.1 & Sacroiliitis, not elsewhere classified \\
\hline M53.2x8 & Spinal instabilities, sacral and sacrococcygeal region \\
\hline M53.3 & Dislocation of sacroiliac and sacrococcygeal joint \\
\hline S33.2xxA & Disorders of sacrum \\
\hline S33.6xxA & Sprain of sacroiliac joint \\
\hline $\mathbf{0 9 9 . 8 9}$ & Other specified diseases and conditions complicating preg- \\
nancy,
\end{tabular}


- Performance of a fluoroscopically-guided SIJ block on the affected side (or both sides, see discussion above) which shows at least a 50\% acute reduction in pain;

- A course of conservative treatment to include use of non-steroidal anti-inflammatory drugs and one of the following: (1) an adequate period of rest, (2) an adequate course of physical therapy wherein the physical therapist specifically documents lack of response to treatment

- SIJ pain has continued for a minimum of six months; and

- All other diagnoses that could be causing the patient's pain have been considered and the physician believes that SIJ fusion is clinically required.

\section{Surgeon Qualifications}

- Minimally invasive SIJ fusion is a surgical procedure performed by orthopedic or neurologic surgeons who have successfully completed a residency in that specialty as well as at least one specialized training course in the procedure. Training should include device placement in cadavers under supervision of a surgeon experienced in the procedure. - Surgeons performing minimally invasive SIJ fusion should be specifically credentialed and/or privileged by at least one hospital to perform the procedure.

\section{Coverage/Conclusion}

The utilization of minimally invasive surgical approach for SIJ fusion has become a recognized safe, predictable and preferred surgical method for the management of intractable, debilitating primary or secondary SIJ pain disorders. ${ }^{57}$

The ISASS policy does not endorse any specific MIS SIJ System. There are numerous devices available that have received FDA $510(\mathrm{k})$ clearance for use in minimally invasive/percutaneous sacroiliac joint fusion stabilization. The instrumentation utilized in a MIS SIJ procedure is the purview of surgeon preference. $^{58}$ 
Table 2. Published literature on minimally invasive SIJ fusion.

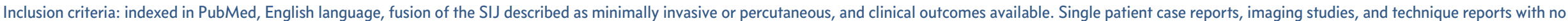
clinical outcomes are excluded. When multiple reports of the same cohort were published, only the most recent (longest follow-up) publication is summarized.

\begin{tabular}{|c|c|c|c|c|c|c|c|}
\hline Author, Year & Study design & $\mathrm{N}$ & Implant & Technique & $\begin{array}{l}\text { Demographics } \\
\text { Mean ( } \pm \text { SD) or } \\
\text { (range), unless } \\
\text { otherwise } \\
\text { specified }\end{array}$ & $\begin{array}{l}\text { Results } \\
\text { Mean ( } \pm \text { SD) or (range) unless } \\
\text { otherwise specified }\end{array}$ & Complications (n) \\
\hline Sturesson $2016^{18}$ & $\begin{array}{l}\text { Prospective, } \\
\text { multicenter, } \\
\text { randomized } \\
\text { controlled trial } \\
\text { (Only surgical } \\
\text { arm reported } \\
\text { herein) } \\
\text { (iMIA, } \\
\text { ClinicalTrials.gov } \\
\text { NCT01741025) }\end{array}$ & 52 & $\begin{array}{l}\text { iFuse } \\
\text { Implant } \\
\text { System }\end{array}$ & $\begin{array}{l}\text { Lateral } \\
\text { approach }\end{array}$ & $\begin{array}{l}\text { Age: } 49.4(27-70) \\
\text { years } \\
\text { Sex: } 38 \mathrm{~F} / 14 \mathrm{M} \\
\text { Prior lumbar } \\
\text { fusion: } 34.6 \% \\
\text { Follow-up: } 6 \mathrm{mo}\end{array}$ & $\begin{array}{l}\text { LBP VAS: } 77.7 \text { pre-op, } 34.4 \text { at } \\
6 \text { mo for an improvement of } 43.3 \\
(25.0) \\
\text { ODI: } 56.6 \text { pre-op; improvement } \\
\text { of } 25.5 \text { at } 6 \mathrm{mo} \\
90 \% \text { very or somewhat satisfied } \\
80 \% \text { would definitely have the } \\
\text { surgery again } \\
\text { Surgical time: } \\
54 \text { (19-107) min } \\
\text { Fluoroscopy time: } 2.1 \text { (1.0-4.0) } \\
\text { min } \\
\text { Hospital stay: } 3 \text { (range } 1-28 \text { ) days }\end{array}$ & $\begin{array}{l}\text { Within } 180 \text { days: } 10 \text { AEs in } 9 \text { subjects ( } 0.19 \text { events per subject), } 8 \text { severe AEs: } \\
\text { device-related }(0) \text {, procedure-related (2, both resolved). } \\
\text { Device- and procedure-related events: postop radicular pain resulting from implant } \\
\text { protrusion into foramen ( } 1 \text {, resolved), postop hematomas ( } 2 \text {, resolved). } \\
\text { No subject has undergone late revision of implants. }\end{array}$ \\
\hline \multirow{2}{*}{$\begin{array}{l}\text { Polly } 2015^{19} \\
\text { (prior pubs from same cohort/ } \\
\text { trial: Whang } 2015-6 \mathrm{mo} \\
\text { results }^{51} \text { ) }\end{array}$} & \multirow{2}{*}{$\begin{array}{l}\text { Prospective, } \\
\text { multicenter, } \\
\text { randomized } \\
\text { controlled trial } \\
\text { (Only surgical } \\
\text { arm reported } \\
\text { herein) } \\
\text { (INSITE, } \\
\text { ClinicalTrials.gov } \\
\text { NCT01681004) }\end{array}$} & 102 & $\begin{array}{l}\text { iFuse } \\
\text { Implant } \\
\text { System }\end{array}$ & $\begin{array}{l}\text { Lateral } \\
\text { approach }\end{array}$ & $\begin{array}{l}\text { Age: } 50.2(26-72) \\
\text { years } \\
\text { Sex: } 75 \mathrm{~F} / 27 \mathrm{M} \\
\text { Prior lumbar } \\
\text { fusion: } 39 \% \\
\text { Follow-up: } 12 \mathrm{mo}\end{array}$ & $\begin{array}{l}\text { VAS: } 82.3(11.9) \text { pre-op, } 28.3 \\
(29.3) \text { at } 12 \mathrm{mo} \\
\text { ODI: } 57.2(12.8) \text { pre-op, } 28.1 \\
(20.8) \text { at } 12 \mathrm{mo} \\
\text { Surgical time: } 44.9(22.3) \mathrm{min} \\
\text { Fluoroscopy time: } 2.5(3.6) \mathrm{min} \\
\text { EBL: } 32.7(32.8) \mathrm{mL} \\
\text { Hospital stay: } 0.8 \text { (range } 0-7) \\
\text { days }\end{array}$ & $\begin{array}{l}\text { Procedure-related adverse events within the first } 6 \mathrm{mo} \text { ( } 180 \text { days): } \\
\text { neuropathic symptoms (2), postoperative medical problems (4: urinary retention, } \\
\text { nausea/vomiting, atrial fibrillation), SIJ pain or trochanteric bursitis (4), surgical } \\
\text { wound problems (4), iliac fracture (1), asymptomatic physical examination } \\
\text { finding (1) }\end{array}$ \\
\hline & & $\begin{array}{l}35 \text { of } \\
44 \\
\text { (NSM patients } \\
\text { that crossed over } \\
\text { after } \\
6 \text { mo visit) }\end{array}$ & $\begin{array}{l}\text { iFuse } \\
\text { Implant } \\
\text { System }\end{array}$ & $\begin{array}{l}\text { Lateral } \\
\text { approach }\end{array}$ & $\begin{array}{l}\text { Age: } 53.0(11.5) \\
\text { years } \\
\text { Sex: } 20 \mathrm{~F} / 15 \mathrm{M} \\
\text { Prior lumbar } \\
\text { fusion: } 39 \% \\
\text { Follow-up: } 6 \mathrm{mo} \\
\text { post-fusion }\end{array}$ & $\begin{array}{l}\text { VAS: } 83.9 \text { pre- op, } 35.8 \text { at } \\
6 \text { mo post MIS SIJ fusion } \\
\text { ODI: } 58.3 \text { pre- op, } 30.2 \text { at } \\
6 \text { mo post MIS } \\
\text { SIJ fusion }\end{array}$ & \\
\hline
\end{tabular}

Downloaded from http://ijssurgery.com/ by guest on April 26, 2023 


\begin{tabular}{|c|c|c|c|c|c|c|c|}
\hline Author, Year & Study design & $\mathrm{N}$ & Implant & Technique & $\begin{array}{l}\text { Demographics } \\
\text { Mean }( \pm \text { SD) or } \\
\text { (range), unless } \\
\text { otherwise } \\
\text { specified }\end{array}$ & $\begin{array}{l}\text { Results } \\
\text { Mean ( } \pm \text { SD) or (range) unless } \\
\text { otherwise specified }\end{array}$ & Complications (n) \\
\hline $\begin{array}{l}\text { Duhon } 2016^{31} \\
\text { (Prior pubs from same cohort/ } \\
\text { trial: Duhon } 2015-12 \mathrm{mo} \\
\text { results } \\
\text { interim results } 2013-6 \mathrm{mo} \\
\text { is }\end{array}$ & $\begin{array}{l}\text { Prospective, } \\
\text { multicenter } \\
\text { (SIFI, } \\
\text { ClinicalTrials.gov } \\
\text { NCT01640353) }\end{array}$ & 172 & $\begin{array}{l}\text { iFuse } \\
\text { Implant } \\
\text { System }\end{array}$ & $\begin{array}{l}\text { Lateral } \\
\text { approach }\end{array}$ & $\begin{array}{l}\text { Age: } 50.9(24-72) \\
\text { years } \\
\text { Sex: } 120 \mathrm{~F} / 52 \mathrm{M} \\
\text { Prior lumbar } \\
\text { fusion: } 44 \% \\
\text { Follow-up: } 24 \mathrm{mo}\end{array}$ & $\begin{array}{l}\text { VAS SI joint pain: } 79.8(12.8) \\
\text { pre-op, } 30.4(27.6) \text { at } 12 \mathrm{mo}, 26.0 \\
(26.7) \text { at } 24 \mathrm{mo} \\
\text { ODI: } 55.2(11.5) \text { pre-op, } 31.5 \\
(19.2) \text { at } 12 \mathrm{mo}, 30.9(20.5) \text { at } \\
24 \mathrm{mo} \\
\text { SF-36 PCS: } 31.7(5.6) \text { pre-op, } \\
40.5(9.6) \text { at } 12 \mathrm{mo}, 40.7(10.3) \text { at } \\
24 \mathrm{mo} \\
\text { SF-36 MCS: } 38.5(11.3) \text { pre-op, } \\
48.2(12.3) \text { at } 12 \mathrm{mo}, 49.0(11.5) \text { at } \\
24 \mathrm{mo} \\
\text { EQ-5D TTO: } 0.43(0.18) \text { pre-op, } \\
0.71(0.20) \text { at } 12 \mathrm{mo}, 0.71(0.22) \text { at } \\
24 \mathrm{mo} \\
\text { Surgical time: } 46.6(16.1) \mathrm{min} \\
\text { Fluoroscopy time: } 2.7(1.8) \mathrm{min} \\
\text { EBL: } 51.0(75.8) \mathrm{mL} \\
\text { Hospital stay: median } 1 \text { (range } \\
0-7) \text { day }\end{array}$ & $\begin{array}{l}\text { Device-related: } \\
\text { Neuropathic pain related to device malposition (3), SI joint or buttock pain (2), SI } \\
\text { joint pain after fall associated with inadequate device placement (1), Hip pain } \\
\text { related to periosteal bone growth around implant (1) } \\
\text { Procedure-related: } \\
\text { Wound drainage/irritation/infection (6), SI joint pain (5), SI joint pain (inadequate } \\
\text { stabilization) (3), implant impingement (3), nausea/vomiting (3), buttock pain (2), } \\
\text { foot weakness related to anesthesia (1), urinary retention (1), vascular injury (1), } \\
\text { wound numbness (1) }\end{array}$ \\
\hline Capobianco $2015^{54}$ & $\begin{array}{l}\text { Prospective, } \\
\text { multicenter } \\
\text { (SIFI, } \\
\text { ClinicalTrials.gov } \\
\text { NCT01640353) } \\
\text { Subsets }\end{array}$ & $\begin{array}{l}20 \\
\text { (Females with } \\
\text { PPGP) }\end{array}$ & $\begin{array}{l}\text { iFuse } \\
\text { Implant } \\
\text { System }\end{array}$ & $\begin{array}{l}\text { Lateral } \\
\text { approach }\end{array}$ & $\begin{array}{l}\text { Age: } 43.3(9.0) \\
\text { years } \\
\text { Sex: } 20 \mathrm{~F} \\
\text { Prior lumbar } \\
\text { fusion: } 30 \% \\
\text { Follow-up: } 12 \mathrm{mo}\end{array}$ & $\begin{array}{l}\text { VAS SI joint pain: } 81.9(10.0) \\
\text { pre-op, } 21.3(17.6) \text { at } 6 \mathrm{mo}, 31.4 \\
(30.9) \text { at } 12 \mathrm{mo} \\
\text { ODI: } 52.2(12.7) \text { pre-op, } 30.4 \\
(20.0) \text { at } 6 \mathrm{mo}, 32.8(21.4) \text { at } \\
12 \mathrm{mo} \\
\text { SF-36 PCS: } 32.0(5.6) \text { pre-op, } \\
40.0(11.1) \text { at } 6 \mathrm{mo}, 41.6(10.8) \text { at } \\
12 \mathrm{mo} \\
\text { SF-36 MCS: } 42.2(12.4) \text { pre-op, } \\
49.7(9.6) \text { at } 6 \mathrm{mo}, 49.0(10.8) \text { at } \\
12 \mathrm{mo} \\
\text { EQ-5D TTO: } 0.42(0.14) \text { pre-op, } \\
0.72(0.23) \text { at } 6 \mathrm{mo}, 0.72(0.21) \text { at } \\
12 \mathrm{mo} \\
100 \% \text { very or somewhat satisfied }\end{array}$ & $\begin{array}{l}37 \text { total adverse events (1.8 event rate per subject) } \\
4 \text { device/procedure-related: wound infection (2), numbness around wound (1), fall } \\
\text { causing SI joint pain (1) }\end{array}$ \\
\hline
\end{tabular}




\begin{tabular}{|c|c|c|c|c|c|c|c|}
\hline Author, Year & Study design & $\mathrm{N}$ & Implant & Technique & $\begin{array}{l}\text { Demographics } \\
\text { Mean ( } \pm \text { SD) or } \\
\text { (range), unless } \\
\text { otherwise } \\
\text { specified }\end{array}$ & $\begin{array}{l}\text { Results } \\
\text { Mean }( \pm \text { SD) or (range) unless } \\
\text { otherwise specified }\end{array}$ & Complications (n) \\
\hline & & $\begin{array}{l}100 \\
\text { (Females with No } \\
\text { PPGP) }\end{array}$ & $\begin{array}{l}\text { iFuse } \\
\text { Implant } \\
\text { System }\end{array}$ & $\begin{array}{l}\text { Lateral } \\
\text { approach }\end{array}$ & $\begin{array}{l}\text { Age: } 52.5(11.1) \\
\text { years } \\
\text { Sex: } 100 \mathrm{~F} \\
\text { Prior lumbar } \\
\text { fusion: } 42.2 \% \\
\text { Follow-up: } 12 \mathrm{mo}\end{array}$ & $\begin{array}{l}\text { VAS SI joint pain: } 79.9(13.3) \\
\text { pre-op, } 31.5(27.0) \text { at } 6 \mathrm{mo}, 32.7 \\
(28.5) \text { at } 12 \mathrm{mo} \\
\text { ODI: } 55.0(11.2) \text { pre-op, } 31.0 \\
(18.7) \text { at } 6 \mathrm{mo}, 30.8(19.1) \text { at } \\
12 \mathrm{mo} \\
\text { SF-36 PCS: } 31.1(5.6) \text { pre-op, } \\
40.5(9.2) \text { at } 6 \mathrm{mo}, 40.0(9.6) \text { at } \\
12 \mathrm{mo} \\
\text { SF-36 MCS: } 37.7(11.6) \text { pre-op, } \\
48.8(10.8) \text { at } 6 \mathrm{mo}, 47.7(12.9) \text { at } \\
12 \mathrm{mo} \\
\text { EQ-5D TTO: } 0.43(0.18) \text { pre-op, } \\
0.70(0.19) \text { at } 6 \mathrm{mo}, 0.70(0.20) \text { at } \\
12 \mathrm{mo} \\
84 \% \text { very or somewhat satisfied }\end{array}$ & $\begin{array}{l}158 \text { total adverse events ( } 1.6 \text { event rate per subject) } \\
10 \text { device/procedure-related: buttock pain (2), post-op neuropathy (1), post-op } \\
\text { nausea/vomiting (3), intraop hemorrhage (1), neuropathy after contralateral SIJ } \\
\text { fusion revision (1), urinary retention (1), would drainage (1) }\end{array}$ \\
\hline & & $\begin{array}{l}52 \\
\text { (Men) }\end{array}$ & $\begin{array}{l}\text { iFuse } \\
\text { Implant } \\
\text { System }\end{array}$ & $\begin{array}{l}\text { Lateral } \\
\text { approach }\end{array}$ & $\begin{array}{l}\text { Age: } 50.7(11.4) \\
\text { years } \\
\text { Sex: } 52 \mathrm{M} \\
\text { Prior lumbar } \\
\text { fusion: } 51.6 \% \\
\text { Follow-up: } 12 \mathrm{mo}\end{array}$ & $\begin{array}{l}\text { VAS SI joint pain: } 78.9(12.9) \\
\text { pre-op, } 30.2(28.0) \text { at } 6 \mathrm{mo}, 25.0 \\
(24.0) \text { at } 12 \mathrm{mo} \\
\text { ODI: } 56.7(11.5) \text { pre-op, } 36.4 \\
(21.4) \text { at } 6 \mathrm{mo}, 31.9(18.9) \text { at } \\
12 \mathrm{mo} \\
\text { SF-36 PCS: } 32.7(5.5) \text { pre-op, } \\
39.8(10.1) \text { at } 6 \mathrm{mo}, 40.5(8.9) \text { at } \\
12 \mathrm{mo} \\
\text { SF-36 MCS: } 38.6(10.3) \text { pre-op, } \\
45.1(13.2) \text { at } 6 \mathrm{mo}, 48.0(12.1) \text { at } \\
12 \mathrm{mo} \\
\text { EQ-5D TTO: } 0.45(0.19) \text { pre-op, } \\
0.64(0.25) \text { at } 6 \mathrm{mo}, 0.72(0.19) \text { at } \\
12 \mathrm{mo} \\
91.3 \% \text { very or somewhat satisfied }\end{array}$ & $\begin{array}{l}88 \text { total adverse events (1.7 event rate per subject) } \\
7 \text { device/procedure-related: wound infection (2), buttock pain (1), post-op } \\
\text { neuropathy (1), SI joint pain (2), staple irritation (1) }\end{array}$ \\
\hline Vanaclocha $2014^{30}$ & $\begin{array}{l}\text { Single center case } \\
\text { series }\end{array}$ & 24 & $\begin{array}{l}\text { iFuse } \\
\text { Implant } \\
\text { System }\end{array}$ & $\begin{array}{l}\text { Lateral } \\
\text { approach }\end{array}$ & $\begin{array}{l}\text { Age: } 47.4(32- \\
71) \text { years } \\
\text { Sex: } 15 \mathrm{~F} / 9 \mathrm{M} \\
\text { Prior lumbar } \\
\text { fusion: } 2 \\
\text { Follow-up: } 23 \text { mo } \\
\text { (1-4.5 years) }\end{array}$ & $\begin{array}{l}\text { VAS: } 8.7 \text { pre-op, } 1.7 \text { at } 1 \mathrm{yr}, 2.1 \text { at } \\
4.5 \mathrm{yrs} \\
\text { ODI: } 54.1 \text { pre- op, } 14.3 \text { at } 1 \mathrm{yr}, \\
16.3 \text { at } 4.5 \mathrm{yrs} \\
\text { Surgical time: } 48 \text { (range } 40-65 \text { ) } \\
\text { min } \\
\text { EBL: } 58 \text { (range } 40-70 \text { ) mL }\end{array}$ & Immediate post-op pain (4-resolved), temporary post-op radicular pain (2) \\
\hline
\end{tabular}

Downloaded from http://ijssurgery.com/ by guest on April 26, 2023 


\begin{tabular}{|c|c|c|c|c|c|c|c|}
\hline Author, Year & Study design & $\mathrm{N}$ & Implant & Technique & $\begin{array}{l}\text { Demographics } \\
\text { Mean }( \pm \mathrm{SD}) \text { or } \\
\text { (range), unless } \\
\text { otherwise } \\
\text { specified }\end{array}$ & $\begin{array}{l}\text { Results } \\
\text { Mean ( } \pm \text { SD) or (range) unless } \\
\text { otherwise specified }\end{array}$ & Complications (n) \\
\hline Rudolf $2014^{33}$ & $\begin{array}{l}\text { Single center case } \\
\text { series }\end{array}$ & 17 & $\begin{array}{l}\text { iFuse } \\
\text { Implant } \\
\text { System }\end{array}$ & $\begin{array}{l}\text { Lateral } \\
\text { approach }\end{array}$ & $\begin{array}{l}\text { Age: } 58(36-85) \\
\text { years } \\
\text { Sex: } 13 \mathrm{~F} / 4 \mathrm{M} \\
\text { Prior lumbar } \\
\text { fusion: } 8 \text { ( } 47 \%) \\
\text { Follow-up: } 60 \mathrm{mo} \\
\text { Bridging bone: } \\
\text { 87\% (13/15) }\end{array}$ & $\begin{array}{l}\text { VAS: } 8.3(1.4) \text { pre-op, } 3.4(2.4) \text { at } \\
\text { 1yr, } 1.4(2.6) \text { at } 2 \mathrm{yrs}, 2.4(2.2) \text { at } \\
\text { 5yrs } \\
\text { ODI: } 21.5(22.7) \text { at } 5 \mathrm{yrs} \\
\text { Surgical time: } 65(18) \mathrm{min}\end{array}$ & $\begin{array}{l}\text { No intraoperative complications, hematoma (1), cellulitis (2), deep wound infection } \\
\text { secondary to diverticulitis (1) }\end{array}$ \\
\hline Sachs $2014^{32}$ & $\begin{array}{l}\text { Multicenter, } \\
\text { retrospective }\end{array}$ & 144 & $\begin{array}{l}\text { iFuse } \\
\text { Implant } \\
\text { System }\end{array}$ & $\begin{array}{l}\text { Lateral } \\
\text { approach }\end{array}$ & $\begin{array}{l}\text { Age: } 58(30-89) \\
\text { years } \\
\text { Sex: } 30 \mathrm{~F} / 10 \mathrm{M} \\
\text { Prior lumbar } \\
\text { fusion: } 62 \% \\
\text { Follow-up: } 16 \\
(12-26) \text { mo }\end{array}$ & $\begin{array}{l}\text { VAS: } 8.6 \text { pre-op, } 2.7 \text { at follow-up } \\
91 \% \text { Very or somewhat satisfied } \\
91.7 \% \text { would have surgery again } \\
\text { Surgical time: } 73 \mathrm{~min} \\
\text { EBL: } 31 \mathrm{~mL} \\
\text { Hospital stay: } 0.8 \text { days }\end{array}$ & $\begin{array}{l}\text { No intraoperative complications. } \\
28 \text { post-op complications, most common: fall (5), trochanteric bursitis (4), } \\
\text { piriformis syndrome (3), facet pain (3). } \\
1 \text { implant revision (1-year revision rate } 0.7 \% \text { ), }\end{array}$ \\
\hline Sachs $2013^{55}$ & $\begin{array}{l}\text { Single center, } \\
\text { retrospective case } \\
\text { series }\end{array}$ & 40 & $\begin{array}{l}\text { iFuse } \\
\text { Implant } \\
\text { System }\end{array}$ & $\begin{array}{l}\text { Lateral } \\
\text { approach }\end{array}$ & $\begin{array}{l}\text { Age: } 58(30-81) \\
\text { years } \\
\text { Sex: } 30 \mathrm{~F} / 10 \mathrm{M} \\
\text { Prior lumbar } \\
\text { fusion: } 30 \% \\
\text { Follow-up: } 12 \text { mo }\end{array}$ & $\begin{array}{l}\text { VAS: } 8.7(1.5) \text { pre-op, } 0.9(1.6) \text { at } \\
12 \mathrm{mo} \\
98 \% \text { reached MCID } \\
100 \% \text { patient satisfaction }\end{array}$ & Piriformis syndrome (1), new LBP (1), facet joint pain (8), trochanteric bursitis (2) \\
\hline Cummings $2013^{43}$ & $\begin{array}{l}\text { Single center, } \\
\text { retrospective case } \\
\text { series }\end{array}$ & 18 & $\begin{array}{l}\text { iFuse } \\
\text { Implant } \\
\text { System }\end{array}$ & $\begin{array}{l}\text { Lateral } \\
\text { approach }\end{array}$ & $\begin{array}{l}\text { Age: } 64(39-81) \\
\text { years } \\
\text { Sex: } 12 \mathrm{~F} / 6 \mathrm{M} \\
\text { Prior lumbar } \\
\text { fusion: } 61 \% \\
\text { Follow-up: } 12 \text { mo }\end{array}$ & $\begin{array}{l}\text { VAS: } 8.9(1.9) \text { pre-op, } 2.3(2.1) \text { at } \\
12 \mathrm{mo} \\
90 \% \text { reached MCID } \\
\text { ODI: } 52.6(18.8) \text { pre-op, } 13.2 \\
(12.6) \text { at } 12 \mathrm{mo} \\
\text { SF-12 PCS: } 37.8 \text { (10.4) pre-op, } \\
44.6(10.5) \text { at } \\
12 \mathrm{mo}\end{array}$ & $\begin{array}{l}\text { Trochanteric bursitis (3), hematoma (1), fluid retention (1), toe numbness (1), } \\
\text { implant malposition (1) }\end{array}$ \\
\hline Gaetani $2013^{29}$ & $\begin{array}{l}\text { Single center, } \\
\text { retrospective case } \\
\text { series }\end{array}$ & 10 & $\begin{array}{l}\text { iFuse } \\
\text { Implant } \\
\text { System }\end{array}$ & $\begin{array}{l}\text { Lateral } \\
\text { approach }\end{array}$ & $\begin{array}{l}\text { Age: } 53.2(36-71) \\
\text { years } \\
\text { Sex: } 12 \text { F Prior } \\
\text { lumbar fusion: } \\
8.3 \% \\
\text { Follow-up: } 10 \\
(8-18) \text { mo }\end{array}$ & $\begin{array}{l}\text { VAS: } 7.7 \text { (1.3) pre-op, } 3(1.2) \text { at } \\
\text { follow-up } \\
\text { ODI: } 31.4 \text { (6.3) pre-op, } 12 \text { (3.5) at } \\
\text { follow-up } \\
\text { RDQ: } 17.6 \text { (1) pre-op, } 3 \text { (4.1) at } \\
\text { follow-up } \\
\text { Surgical time: } 65 \text { (16) min } \\
\text { EBL: <45 mL } \\
3 \text { month CT scans show initial } \\
\text { fusion }\end{array}$ & Local hematoma (2), low back pain (1) \\
\hline
\end{tabular}




\begin{tabular}{|c|c|c|c|c|c|c|c|}
\hline Author, Year & Study design & $\mathrm{N}$ & Implant & Technique & $\begin{array}{l}\text { Demographics } \\
\text { Mean ( } \pm \text { SD) or } \\
\text { (range), unless } \\
\text { otherwise } \\
\text { specified }\end{array}$ & $\begin{array}{l}\text { Results } \\
\text { Mean ( } \pm \text { SD) or (range) unless } \\
\text { otherwise specified }\end{array}$ & Complications (n) \\
\hline Schroeder $2013^{44}$ & $\begin{array}{l}\text { Single center, } \\
\text { retrospective case } \\
\text { series }\end{array}$ & 6 & $\begin{array}{l}\text { iFuse } \\
\text { Implant } \\
\text { System }\end{array}$ & $\begin{array}{l}\text { Lateral } \\
\text { approach }\end{array}$ & $\begin{array}{l}\text { Age: } 50(25-60) \\
\text { years } \\
\text { Sex: } 6 \mathrm{~F} / 0 \mathrm{M} \text { Prior } \\
\text { lumbar fusion: } \\
\text { 100\% (deformity } \\
\text { correction) } \\
\text { Follow-up: } 10.25 \\
\text { (4-15) mo }\end{array}$ & $\begin{array}{l}\text { VAS: } 7.83 \text { pre- op, } 2.67 \text { at } \\
\text { follow-up } \\
\text { ODI: } 22.1 \text { pre- op, } 10.5 \text { at } \\
\text { follow-up } \\
\text { Hospital stay: } 2 \text { days (range } 1-4 \text { ) } \\
\text { Bony bridging seen in } 4 \text { patients }\end{array}$ & No intraoperative or post-operative complications. \\
\hline \multirow{4}{*}{ Rudolf $2013^{41}$} & \multirow{4}{*}{$\begin{array}{l}\text { Single center, } \\
\text { sub-group } \\
\text { analysis }\end{array}$} & 40 & $\begin{array}{l}\text { iFuse } \\
\text { Implant } \\
\text { System }\end{array}$ & $\begin{array}{l}\text { Lateral } \\
\text { approach }\end{array}$ & \multicolumn{3}{|c|}{ Subgroup analysis from Rudolf 2012 to assess effect of prior lumbar fusion or lumbar treatment on outcomes. Follow up: 12 and 24 months } \\
\hline & & $\begin{array}{l}18 \text { (no prior } \\
\text { fusion) }\end{array}$ & $\begin{array}{l}\text { iFuse } \\
\text { Implant } \\
\text { System }\end{array}$ & $\begin{array}{l}\text { Lateral } \\
\text { approach }\end{array}$ & $\begin{array}{l}\text { Age: } 49(12) \\
\text { Sex: 12F/6M }\end{array}$ & $\begin{array}{l}\text { VAS decrease at } 12 \mathrm{mo}:-5.94 \\
(3.3) \\
\text { VAS decrease at } 24 \mathrm{mo}:-5.47 \\
(2.88) \\
\text { Surgical time: } 60(19) \mathrm{min}\end{array}$ & Superficial cellulitis (2), wound infection (1), revision for implant malposition (1) \\
\hline & & 15 (prior fusion) & $\begin{array}{l}\text { iFuse } \\
\text { Implant } \\
\text { System }\end{array}$ & $\begin{array}{l}\text { Lateral } \\
\text { approach }\end{array}$ & $\begin{array}{l}\text { Age: } 58(11) \\
\text { Sex: } 11 F / 4 M\end{array}$ & $\begin{array}{l}\text { VAS decrease at } 12 \mathrm{mo}: \\
-3.50(3.46) \\
\text { VAS decrease at } 24 \mathrm{mo}: \\
-5.81(2.88) \\
\text { Surgical time: } 64(19) \mathrm{min}\end{array}$ & Superficial cellulitis (2), buttock hematoma (1), revision for implant malposition (1) \\
\hline & & $\begin{array}{l}7 \text { (prior } \\
\text { concomitant } \\
\text { lumbar pathology } \\
\text { treated } \\
\text { non-surgically }\end{array}$ & $\begin{array}{l}\text { iFuse } \\
\text { Implant } \\
\text { System }\end{array}$ & $\begin{array}{l}\text { Lateral } \\
\text { approach }\end{array}$ & $\begin{array}{l}\text { Age: } 58(17) \\
\text { Sex: } 3 F / 4 M\end{array}$ & $\begin{array}{l}\text { VAS decrease at } 12 \mathrm{mo}: \\
-3.71(3.11) \\
\text { VAS decrease at } 24 \mathrm{mo}: \\
-4.79(4.28) \\
\text { Surgical time: } 64(19) \mathrm{min}\end{array}$ & None \\
\hline Endres $2013^{38}$ & $\begin{array}{l}\text { Single center, } \\
\text { Retrospective } \\
\text { case series }\end{array}$ & 19 & $\begin{array}{l}\text { DIANA } \\
\text { cage } \\
\text { [Product } \\
\text { not } \\
\text { approved } \\
\text { for use in } \\
\text { the US] }\end{array}$ & $\begin{array}{l}\text { Posterior, } \\
\text { longitudinally } \\
\text { inserted into } \\
\text { SI joint }\end{array}$ & $\begin{array}{l}\text { Age: } 60.9(36-76) \\
\text { years } \\
\text { Sex: } 5 \mathrm{~F} / 14 \mathrm{M} \\
\text { Prior lumbar } \\
\text { fusion: } 100 \% \\
\text { Follow-up: } 13.2 \\
(6-24) \text { mo }\end{array}$ & $\begin{array}{l}\text { VAS: } 8.5(7.5-9) \text { pre-op to } \\
6.0(2.2-9) \text { at follow-up } \\
\text { ODI: } 64.1 \text { ( } 40-82) \text { pre-op to } 56.97 \\
(8-82) \text { at follow-up } \\
\text { EBL: }<150 \mathrm{~mL} \\
\text { Hospital stay: } 7.3(3-10) \text { days } \\
\text { Fusion rate: } 78.9 \%(15 / 19 \text { joints), } \\
\text { defined as lack of loosening and } \\
\text { evidence of bone bridging around } \\
\text { the implant }\end{array}$ & No neurovascular complications \\
\hline
\end{tabular}




\begin{tabular}{|c|c|c|c|c|c|c|c|}
\hline Author, Year & Study design & $\mathrm{N}$ & Implant & Technique & $\begin{array}{l}\text { Demographics } \\
\text { Mean ( } \pm \text { SD) or } \\
\text { (range), unless } \\
\text { otherwise } \\
\text { specified }\end{array}$ & $\begin{array}{l}\text { Results } \\
\text { Mean ( } \pm \text { SD) or (range) unless } \\
\text { otherwise specified }\end{array}$ & Complications (n) \\
\hline Mason $2013^{36}$ & $\begin{array}{l}\text { Retrospective } \\
\text { case series }\end{array}$ & 55 & $\begin{array}{l}\text { HMA } \\
\text { screw } \\
\text { packed } \\
\text { with DBM }\end{array}$ & $\begin{array}{l}\text { Lateral } \\
\text { approach }\end{array}$ & $\begin{array}{l}\text { Age: } 57 \text { years } \\
\text { Sex: } 46 \mathrm{~F} / 9 \mathrm{M} \\
\text { Prior lumbar } \\
\text { fusion: } 40 \% \\
\text { Follow-up: } 36 \\
(12-84) \text { mo }\end{array}$ & $\begin{array}{l}\text { VAS: } 8.05 \text { (1.9) pre-op, } 4.48 \\
\text { (2.81) at follow-up } \\
\text { SF-36PCS: } 26.6 \text { (15.2) pre-op, } 43 \\
\text { (22.68) follow-up } \\
\text { Majeed scoring: } 36.18 \text { (15.08) } \\
\text { pre- op, } 64.78 \text { (20.18) follow-up }\end{array}$ & Post-op nerve pain requiring reoperation (2) \\
\hline Rudolf $2012^{28}$ & $\begin{array}{l}\text { Single center, } \\
\text { retrospective case } \\
\text { series }\end{array}$ & 50 & $\begin{array}{l}\text { iFuse } \\
\text { Implant } \\
\text { System }\end{array}$ & $\begin{array}{l}\text { Lateral } \\
\text { approach }\end{array}$ & $\begin{array}{l}\text { Age: } 54(24-85) \\
\text { years } \\
\text { Sex: } 34 \mathrm{~F} / 16 \mathrm{M} \\
\text { Prior lumbar } \\
\text { fusion: } 44 \% \\
\text { Follow-up: } 40 \\
\text { (24-56) mo }\end{array}$ & $\begin{array}{l}\text { VAS: } 7.6 \text { pre- op, } 2.0 \text { at follow-up } \\
82 \% \text { reached MCID } \\
82 \% \text { patient satisfaction } \\
\text { Surgical time: } 65 \text { (26) min }\end{array}$ & Superficial cellulitis (3), deep wound infection (1), hematoma (2), reoperation (3) \\
\hline Sachs $2012^{42}$ & $\begin{array}{l}\text { Single center, } \\
\text { retrospective case } \\
\text { series }\end{array}$ & 11 & $\begin{array}{l}\text { iFuse } \\
\text { Implant } \\
\text { System }\end{array}$ & $\begin{array}{l}\text { Lateral } \\
\text { approach }\end{array}$ & $\begin{array}{l}\text { Age: } 65(45-82) \\
\text { years } \\
\text { Sex: } 10 \mathrm{~F} / 1 \mathrm{M} \\
\text { Prior lumbar } \\
\text { fusion: } 18 \% \\
\text { Follow-up: } 12 \text { mo }\end{array}$ & $\begin{array}{l}\text { VAS: } 7.9(2.2) \text { pre-op, } 2.3(3.1) \text { at } \\
\text { 12mo } \\
\text { Surgical time: } 77.5(31.8) \mathrm{min} \\
\text { EBL: } 21.8(18.9) \mathrm{mL}\end{array}$ & Piriformis syndrome (1), low back pain (1) \\
\hline McGuire $2012^{21}$ & $\begin{array}{l}\text { Retrospective } \\
\text { case series }\end{array}$ & 37 & $\begin{array}{l}\text { Fibular } \\
\text { allograft } \\
\text { dowels }\end{array}$ & $\begin{array}{l}\text { Posterior, } \\
\text { longitudinally } \\
\text { inserted into } \\
\text { SI joint }\end{array}$ & $\begin{array}{l}\text { Age: } 42.5(23-63) \\
\text { Years } \\
\text { Sex: } 34 \mathrm{~F} / 3 \mathrm{M} \\
\text { Follow-up: } 39.6 \\
\text { (8-62) mo }\end{array}$ & $\begin{array}{l}\text { Baseline VAS: } 9.1 \\
\text { Final VAS: } 3.4 \\
\text { Fusion rate: } 89.5 \%\end{array}$ & $\begin{array}{l}\text { Nonunion requiring revision (4) } \\
(10.5 \%)\end{array}$ \\
\hline Khurana $2009^{35}$ & $\begin{array}{l}\text { Retrospective } \\
\text { case series }\end{array}$ & 15 & $\begin{array}{l}\text { HMA } \\
\text { screw } \\
\text { packed } \\
\text { with DBM }\end{array}$ & $\begin{array}{l}\text { Lateral } \\
\text { approach }\end{array}$ & $\begin{array}{l}\text { Age: } 48.7 \\
\text { (37.3-62.6) years } \\
\text { Sex: } 11 \mathrm{~F} / 4 \mathrm{M} \\
\text { Prior lumbar } \\
\text { fusion: } 40 \% \\
\text { Follow-up: } 17 \\
\text { (9-39) mo }\end{array}$ & $\begin{array}{l}\text { SF-36 PF: } 37.15(14.28) \text { pre- op, } \\
79.33 \text { (12.52) at follow-up } \\
\text { Majeed's: } 37(18-54) \text { pre- op, } 79 \\
\text { (63-96) at follow-up } \\
\text { Good to excellent results: } \\
13 / 15(87 \%) \\
\text { EBL: }<50 \mathrm{ml} \\
\text { Hospital stay: } 2.7 \text { (1-7) days }\end{array}$ & No post-operative neurological or wound complications. \\
\hline
\end{tabular}




\begin{tabular}{|c|c|c|c|c|c|c|c|}
\hline Author, Year & Study design & $\mathrm{N}$ & Implant & Technique & $\begin{array}{l}\text { Demographics } \\
\text { Mean }( \pm \mathrm{SD}) \text { or } \\
\text { (range), unless } \\
\text { otherwise } \\
\text { specified }\end{array}$ & $\begin{array}{l}\text { Results } \\
\text { Mean ( } \pm \text { SD) or (range) unless } \\
\text { otherwise specified }\end{array}$ & Complications (n) \\
\hline Al-Khayer $2008^{34}$ & $\begin{array}{l}\text { Retrospective } \\
\text { case series }\end{array}$ & 9 & $\begin{array}{l}\text { HMA } \\
\text { screw } \\
\text { packed } \\
\text { with DBM }\end{array}$ & $\begin{array}{l}\text { Lateral } \\
\text { approach }\end{array}$ & $\begin{array}{l}\text { Age: } 42(35-56) \\
\text { years } \\
\text { Sex: } 9 F \\
\text { Follow-up: } 40 \\
\text { (24-70) mo }\end{array}$ & $\begin{array}{l}\text { VAS decreased: } 8.1(7-9) \text { to } 4.6 \\
(3-7) \\
\text { ODI decreased: } 59(34-70) \text { to } 45 \\
\text { (28-60) } \\
\text { EBL: }<50 \mathrm{ml} \\
\text { Hospital stay: } 6.9 \text { (2-11) days } \\
\text { Return to work: } 44.44 \%\end{array}$ & Deep wound infection requiring debridement and IV antibiotics (1) \\
\hline Wise $2008^{37}$ & $\begin{array}{l}\text { Single center } \\
\text { Prospective } \\
\text { cohort }\end{array}$ & 13 & $\begin{array}{l}\text { Titanium } \\
\text { cage } \\
\text { packed } \\
\text { with BMP }\end{array}$ & $\begin{array}{l}\text { Posterior, } \\
\text { Longitudinally } \\
\text { inserted into } \\
\text { SIJ }\end{array}$ & $\begin{array}{l}\text { Age: } 53.1(45-62) \\
\text { years } \\
\text { Sex: } 12 \mathrm{~F} / 1 \mathrm{M} \\
\text { Prior lumbar } \\
\text { fusion: } 61.5 \% \\
\text { Follow-up: } 29.5 \\
\text { (24-35) mo }\end{array}$ & $\begin{array}{l}\text { Back VAS improved by } 4.9 \mathrm{pts} \\
\text { Leg VAS improved by } 2.4 \mathrm{pts} \\
\text { EBL: }<100 \mathrm{ml} \\
\text { Hospital stay: } 1.7 \text { days } \\
\text { Fusion rate: } 89 \% \text { (17/19 joints) on } \\
\text { CT at } 6 \mathrm{mo}\end{array}$ & Reoperation via open arthrodesis secondary to nonunion and persistent pain (1) \\
\hline \multicolumn{8}{|c|}{ Comparative cohort studies of open surgery vs MIS } \\
\hline \multirow{2}{*}{ Ledonio $2014^{45}$} & \multirow{2}{*}{$\begin{array}{l}\text { Single center, } \\
\text { retrospective, } \\
\text { comparative } \\
\text { cohort study }\end{array}$} & 22 & $\begin{array}{l}\text { iFuse } \\
\text { Implant } \\
\text { System }\end{array}$ & $\begin{array}{l}\text { Lateral } \\
\text { approach }\end{array}$ & $\begin{array}{l}\text { MIS Cohort } \\
\text { Age: } 47.9(13.1) \\
\text { years } \\
\text { Sex: } 17 \mathrm{~F} / 5 \mathrm{M} \\
\text { Prior lumbar } \\
\text { fusion: } 64 \% \\
\text { Follow-up: } \\
\text { median } 15 \\
(12-26) \text { mo }\end{array}$ & $\begin{array}{l}\text { ODI: } 61.5(12.5) \text { pre-op, } 52(16.9) \\
\text { at follow-up } \\
\text { Surgical time: } 68.3(26.8) \mathrm{min} \\
\text { EBL: } 40.5(31.4) \mathrm{mL} \\
\text { Hospital Stay: } 2.0(1.5) \text { days }\end{array}$ & $\begin{array}{l}\text { Pulmonary embolism that resolved with treatment (1), revisions due to halo } \\
\text { formation on the sacral side with recurring sacroiliac joint pain (2) }\end{array}$ \\
\hline & & 22 & $\begin{array}{l}3 \text { hole, } \\
4.5 \mathrm{~mm} \\
\text { plate, } \\
\text { autograft } \\
\text { packed } \\
\text { within } \\
\text { joint }\end{array}$ & $\begin{array}{l}\text { Anterior } \\
\text { approach } \\
\text { through an } \\
\text { ilioinguinal } \\
\text { incision }\end{array}$ & $\begin{array}{l}\text { Open Cohort } \\
\text { Age: } 51(9.4) \\
\text { years } \\
\text { Sex:13F/9M } \\
\text { Prior lumbar } \\
\text { fusion: } 50 \% \\
\text { Follow-up: } \\
\text { median 13 } \\
(11-33) \text { mo }\end{array}$ & $\begin{array}{l}\text { ODI: } 61.8(10.8) \text { pre-op, } 47.4 \\
(21.7) \text { at follow-up } \\
\text { Surgical time: } 128(27.9) \mathrm{min} \\
\text { EBL: } 168.8(479.0) \mathrm{mL} \\
\text { Hospital Stay: } 3.3(1.1) \text { days }\end{array}$ & Pulmonary embolism (1), revision due to failed implant and nerve root irritation (2) \\
\hline
\end{tabular}




\begin{tabular}{|c|c|c|c|c|c|c|c|}
\hline Author, Year & Study design & $\mathrm{N}$ & Implant & Technique & $\begin{array}{l}\text { Demographics } \\
\text { Mean ( } \pm \text { SD) or } \\
\text { (range), unless } \\
\text { otherwise } \\
\text { specified }\end{array}$ & $\begin{array}{l}\text { Results } \\
\text { Mean }( \pm \text { SD) or (range) unless } \\
\text { otherwise specified }\end{array}$ & Complications (n) \\
\hline \multirow{2}{*}{ Ledonio $2014^{46}$} & \multirow{2}{*}{$\begin{array}{l}\text { Multicenter, } \\
\text { retrospective, } \\
\text { comparative } \\
\text { cohort study }\end{array}$} & 17 & $\begin{array}{l}\text { iFuse } \\
\text { Implant } \\
\text { System }\end{array}$ & $\begin{array}{l}\text { Lateral } \\
\text { approach }\end{array}$ & $\begin{array}{l}\text { MIS Cohort } \\
\text { Age: median } 66 \\
\text { (39-82) years } \\
\text { Sex: } 11 \mathrm{~F} / 6 \mathrm{M} \\
\text { Prior lumbar } \\
\text { fusion: } 82 \% \\
\text { Follow-up: } 12 \text { mo }\end{array}$ & $\begin{array}{l}\text { Values reported as median } \\
\text { (range) } \\
\text { ODI: } 53 \text { (14-84) pre-op, } 13 \text { (0-38) } \\
\text { at } 12 \text { mo } \\
\text { Surgical time: } 27(18-72) \text { min } \\
\text { Hospital Stay: } 1(1-2) \text { days }\end{array}$ & $\begin{array}{l}\text { Transient trochanteric bursitis (3), hematoma (1), transient toe numbness (1), } \\
\text { revision due to malpositioned implant (1) }\end{array}$ \\
\hline & & 22 & $\begin{array}{l}3 \text { hole, } \\
4.5 \mathrm{~mm} \\
\text { plate, } \\
\text { autograft } \\
\text { packed } \\
\text { within } \\
\text { joint }\end{array}$ & $\begin{array}{l}\text { Anterior } \\
\text { approach } \\
\text { through an } \\
\text { ilioinguinal } \\
\text { incision }\end{array}$ & $\begin{array}{l}\text { Open Cohort } \\
\text { Age: median } 51 \\
(34-74) \text { years } \\
\text { Sex: } 82 \mathrm{~F} / 32 \mathrm{M} \\
\text { Prior lumbar } \\
\text { fusion: } 47 \% \\
\text { Follow-up: } 24 \text { mo }\end{array}$ & $\begin{array}{l}\text { Values reported as median } \\
\text { (range) } \\
\text { ODI: } 64 \text { (44-78) pre-op, } 46 \\
\text { (10-80) at } 12 \text { mo } \\
\text { Surgical time: } 128 \text { (73-180) min } \\
\text { Hospital Stay: } 3 \text { (2-6) days }\end{array}$ & Pulmonary embolism (1), revision due to failed implant and nerve root irritation (2) \\
\hline \multirow{2}{*}{ Graham- Smith $2013^{26}$} & \multirow{2}{*}{$\begin{array}{l}\text { Multicenter, } \\
\text { retrospective } \\
\text { comparative } \\
\text { cohort study }\end{array}$} & 114 & $\begin{array}{l}\text { iFuse } \\
\text { Implant } \\
\text { System }\end{array}$ & $\begin{array}{l}\text { Lateral } \\
\text { approach }\end{array}$ & $\begin{array}{l}\text { MIS Cohort } \\
\text { Age: } 57.4(14.0) \\
\text { years } \\
\text { Sex: } 82 \mathrm{~F} / 32 \mathrm{M} \\
\text { Prior lumbar } \\
\text { fusion: } 47.4 \% \\
\text { Follow-up: } 24 \text { mo }\end{array}$ & $\begin{array}{l}\text { VAS: } 8.3(1.6) \text { pre-op, } 2.3(2.6) \text { at } \\
12 \mathrm{mo}, 1.7(2.9) \text { at } 24 \mathrm{mo} \\
\text { MCID: } 86 \% \text { reached at } 12 \mathrm{mo} \text {, } \\
82 \% \text { at } 24 \mathrm{mo} \\
\text { Surgical time: } 70(24) \mathrm{min} \\
\text { EBL: } 33(27) \mathrm{mL} \\
\text { Hospital stay: } 1.3(0.5) \\
\text { Days }\end{array}$ & $\begin{array}{l}\text { No intraoperative. } \\
\text { Postop repositioning of implants (4), } 3.5 \% \text { (4/114). }\end{array}$ \\
\hline & & 149 & $\begin{array}{l}\text { Screws, } \\
\text { plates }\end{array}$ & $\begin{array}{l}\text { Open } \\
\text { posterior } \\
\text { approach }\end{array}$ & $\begin{array}{l}\text { Open Cohort } \\
\text { Age: } 45.8(11.3) \\
\text { years } \\
\text { Sex: } 103 \mathrm{~F} / 46 \mathrm{M} \\
\text { Prior lumbar } \\
\text { fusion: } 23.5 \% \\
\text { Follow-up: } 24 \text { mo }\end{array}$ & $\begin{array}{l}\text { VAS: } 7.1(1.9) \text { pre-op, } 4.6(3.0) \text { at } \\
12 \mathrm{mo}, 5.6(2.9) \text { at } 24 \mathrm{mo} \\
\text { MCID: } 61 \% \text { reached at } 12 \mathrm{mo} \text {, } \\
50 \% \text { at } 24 \mathrm{mo} \\
\text { Surgical time: } 163(25) \mathrm{min} \\
\text { EBL: } 288(182) \mathrm{mL} \\
\text { Hospital stay: } 5.1(1.9) \\
\text { Days }\end{array}$ & $\begin{array}{l}\text { No intraoperative. } \\
\text { Postop removal of implants (66), } 44 \% \text { (66/149). }\end{array}$ \\
\hline
\end{tabular}

NOTE: The table excludes 3 systematic reviews:

Zaidi - J Neurosurg Spine $2015^{49}$ : systematic review of studies on SIJ fusion, includes open and MIS.

Heiney - Int J Spine Surg 2015"8: systematic review and meta-analysis of MIS SIJ fusion utilizing a lateral transarticular technique.

Lingutla - Eur Spine J $2016^{56}$ : Systematic review and meta-analysis of observational studies describing outcome of SIJ fusion in patients with LBP.

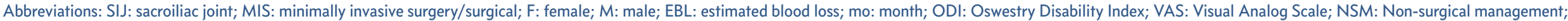
DBM: demineralized bone matrix; HMA: hollow modular anchorage; BMP: bone morphogenic protein. 


\section{References}

1. Szadek KM, Hoogland PV, Zuurmond WW, de Lange JJ, Perez RS. Nociceptive nerve fibers in the sacroiliac joint in humans. Reg Anesth Pain Med. 2008;33(1):36-43.

2. Bernard TN, Kirkaldy-Willis WH. Recognizing specific characteristics of nonspecific low back pain. Clin Orthop. 1987;(217):266-280.

3. Schwarzer AC, Aprill CN, Bogduk N. The sacroiliac joint in chronic low back pain. Spine. 1995;20(1):31-37.

4. Maigne JY, Aivaliklis A, Pfefer F. Results of sacroiliac joint double block and value of sacroiliac pain provocation tests in 54 patients with low back pain. Spine. 1996;21(16):1889-1892.

5. Irwin RW, Watson T, Minick RP, Ambrosius WT. Age, Body Mass Index, and Gender Differences in Sacroiliac Joint Pathology. Am J Phys Med Rehabil. 2007;86(1):37-44.

6. Sembrano JN, Polly DW. How often is low back pain not coming from the back? Spine.

2009;34(1):E27-E32.

7. Szadek KM, van der Wurff P, van Tulder MW, Zuurmond WW, Perez RSGM. Diagnostic validity of criteria for sacroiliac joint pain: a systematic review. J Pain. 2009;10(4):354-368.

8. Cher D, Polly D, Berven S. Sacroiliac Joint pain: burden of disease. Med Devices Evid Res.

2014;7:73-81.

9. Cher DJ, Reckling WC. Quality of life in preoperative patients with sacroiliac joint dysfunction is at least as depressed as in other lumbar spinal conditions. Med Devices Evid Res. 2015;8:395-403.

10. Eno J-J, Boone C, Bellino M, Bishop J. The Prevalence of Sacroiliac Joint Degeneration in Asymptomatic Adults. J Bone Joint Surg Am. 2015;97(11):932-936.

11. Broadhurst NA, Bond MJ. Pain provocation tests for the assessment of sacroiliac joint dysfunction. J Spinal Disord. 1998;11(4):341-345.

12. Polly D, Cher D, Whang PG, Frank C, Sembrano J, for the INSITE Study Group. Does Level of Response to SI Joint Block Predict Response to SI Joint Fusion? Int J Spine Surg. 2016;10:Article 4.

13. Cohen SP, Strassels SA, Kurihara C, et al. Outcome Predictors for Sacroiliac Joint (Lateral Branch)
Radiofrequency Denervation. Reg Anesth Pain Med. 2009;34(3):206-214.

14. Cohen SP, Hurley RW, Buckenmaier CC, Kurihara C, Morlando B, Dragovich A. Randomized placebo-controlled study evaluating lateral branch radiofrequency denervation for sacroiliac joint pain. Anesthesiology. 2008;109(2):279-288.

15. Patel N, Gross A, Brown L, Gekht G. A randomized, placebo-controlled study to assess the efficacy of lateral branch neurotomy for chronic sacroiliac joint pain. Pain Med Malden Mass.

2012;13(3):383-398.

16. Stuge B, Laerum E, Kirkesola G, Vøllestad N. The efficacy of a treatment program focusing on specific stabilizing exercises for pelvic girdle pain after pregnancy: a randomized controlled trial. Spine. 2004;29(4):351-359.

17. Patel N. Twelve-Month Follow-Up of a Randomized Trial Assessing Cooled Radiofrequency Denervation as a Treatment for Sacroiliac Region Pain. Pain Pract Off J World Inst Pain. January 2015. 18. Sturesson B, Kools D, Pflugmacher R, Gasbarrini A, Prestamburgo D, Dengler J. Six-Month Outcomes from a Randomized Controlled Trial of Minimally Invasive SI Joint Fusion vs. Conservative Management. Eur Spine J. 2016; in press.

19. Polly DW, Cher DJ, Wine KD, et al. Randomized Controlled Trial of Minimally Invasive Sacroiliac Joint Fusion Using Triangular Titanium Implants vs Nonsurgical Management for Sacroiliac Joint Dysfunction: 12-Month Outcomes. Neurosurgery. 2015;77(5):674-691.

20. Painter CF. Excision of the os innominatum. Arthrodesis of the sacro-iliac synchrondrosis. Boston Med Surg J. 1908;159(7):205-208.

21. McGuire RA, Chen Z, Donahoe K. Dual fibular allograft dowel technique for sacroiliac joint arthrodesis. Evid-Based Spine-Care J. 2012;3(3):21-28.

22. Buchowski JM, Kebaish KM, Sinkov V, Cohen DB, Sieber AN, Kostuik JP. Functional and radiographic outcome of sacroiliac arthrodesis for the disorders of the sacroiliac joint. Spine J Off J North Am Spine Soc. 2005;5(5):520-528; discussion 529. 23. Belanger TA, Dall BE. Sacroiliac arthrodesis using a posterior midline fascial splitting approach and pedicle screw instrumentation: a new technique. $J$ 
Spinal Disord. 2001;14(2):118-124.

24. Waisbrod H, Krainick JU, Gerbershagen HU. Sacroiliac joint arthrodesis for chronic lower back pain. Arch Orthop Trauma Surg Arch Für Orthop UnfChir. 1987;106(4):238-240.

25. Moore MR. Surgical treatment of chronic painful sacroiliac joint dysfunction. In: Movement, Stability, and Low Back Pain: The Essential Role of the Pelvis. New York: Churchill Livingstone; 1997:563-572.

26. Graham Smith A, Capobianco R, Cher D, et al. Open versus minimally invasive sacroiliac joint fusion: a multi-center comparison of perioperative measures and clinical outcomes. Ann Surg Innov Res. 2013;7(1):14.

27. Sachs D, Capobianco R. Minimally invasive sacroiliac joint fusion: one-year outcomes in $40 \mathrm{pa}-$ tients. Adv Orthop. 2013;2013:536128.

28. Rudolf L. Sacroiliac Joint Arthrodesis-MIS Technique with Titanium Implants: Report of the First 50 Patients and Outcomes. Open Orthop J. 2012;6:495-502.

29. Gaetani P, Miotti D, Risso A, et al. Percutaneous arthrodesis of sacro-iliac joint: a pilot study. $J$ Neurosurg Sci. 2013;57(4):297-301.

30. Vanaclocha VV, Verdú-López F, Sánchez-Pardo, M, et al. Minimally Invasive Sacroiliac Joint Arthrodesis: Experience in a Prospective Series with 24 Patients. J Spine. 2014;3(5).

31. Duhon B, Bitan F, Lockstadt H, Kovalsky D, Cher D, Hillen T. Triangular Titanium Implants for Minimally Invasive Sacroiliac Joint Fusion: 2-Year Follow-Up from a Prospective Multicenter Trial. Int J Spine Surg. 2016;10:Article 13.

32. Sachs D, Capobianco R, Cher D, et al. One-year outcomes after minimally invasive sacroiliac joint fusion with a series of triangular implants: a multicenter, patient-level analysis. Med Devices Evid Res. 2014;7:299-304.

33. Rudolf L, Capobianco R. Five-year clinical and radiographic outcomes after minimally invasive sacroiliac joint fusion using triangular implants. Open Orthop J. 2014;8:375-383.

34. Al-Khayer A, Hegarty J, Hahn D, Grevitt MP. Percutaneous sacroiliac joint arthrodesis: a novel technique. J Spinal Disord Tech. 2008;21(5):359-363. 35. Khurana A, Guha AR, Mohanty K, Ahuja S.
Percutaneous fusion of the sacroiliac joint with hollow modular anchorage screws: clinical and radiological outcome. J Bone Joint Surg Br.

2009;91(5):627-631.

36. Mason LW, Chopra I, Mohanty K. The percutaneous stabilisation of the sacroiliac joint with hollow modular anchorage screws: a prospective outcome study. Eur Spine J. 2013;22(10):2325-2331.

37. Wise CL, Dall BE. Minimally invasive sacroiliac arthrodesis: outcomes of a new technique. J Spinal Disord Tech. 2008;21(8):579-584.

38. Endres S, Ludwig E. Outcome of distraction interference arthrodesis of the sacroiliac joint for sacroiliac arthritis. Indian J Orthop.

2013;47(5):437-442.

39. Cher DJ, Frasco MA, Arnold RJ, Polly DW. Cost-effectiveness of minimally invasive sacroiliac joint fusion. Clin Outcomes Res CEOR. 2016;8:1-14. 40. Rudolf L. Sacroiliac Joint Arthrodesis-MIS Technique with Titanium Implants: Report of the First 50 Patients and Outcomes. Open Orthop J. 2012;6(1):495-502.

41. Rudolf L. MIS Fusion of the SI Joint: Does Prior Lumbar Spinal Fusion Affect Patient Outcomes? Open Orthop J. 2013;7:163-168.

42. Sachs D, Capobianco R. One year successful outcomes for novel sacroiliac joint arthrodesis system. Ann Surg Innov Res. 2012;6(1):13.

43. Cummings J Jr, Capobianco RA. Minimally invasive sacroiliac joint fusion: one-year outcomes in 18 patients. Ann Surg Innov Res. 2013;7(1):12.

44. Schroeder JE, Cunningham ME, Ross T, Boachie-Adjei O. Early Results of Sacro-Iliac Joint Fixation Following Long Fusion to the Sacrum in Adult Spine Deformity. Hosp Spec Surg J. 2013;10(1):30-35.

45. Ledonio CGT, Polly DW, Swiontkowski MF. Minimally invasive versus open sacroiliac joint fusion: are they similarly safe and effective? Clin Orthop. 2014;472(6):1831-1838.

46. Ledonio C, Polly D, Swiontkowski MF, Cummings J. Comparative effectiveness of open versus minimally invasive sacroiliac joint fusion. Med Devices Evid Res. 2014;2014(7):187-193.

47. Cher DJ, Reckling WC, Capobianco RA. Implant survivorship analysis after minimally invasive sacroiliac joint fusion using the iFuse Implant Sys- 
tem. Med Devices Evid Res. 2015;8:485-492.

48. Heiney J, Capobianco R, Cher D. Systematic review of minimally invasive sacroiliac joint fusion using a lateral transarticular approach. Int J Spine Surg. 2015;9:Article 40.

49. Zaidi HA, Montoure AJ, Dickman CA. Surgical and clinical efficacy of sacroiliac joint fusion: a systematic review of the literature. J Neurosurg Spine. April 2015:1-8.

50. Miller L, Reckling WC, Block JE. Analysis of postmarket complaints database for the iFuse SI Joint Fusion System: a minimally invasive treatment for degenerative sacroiliitis and sacroiliac joint disruption. Med Devices Evid Res. 2013;6:77-84.

51. Whang PG, Cher D, Polly D, et al. Sacroiliac Joint Fusion Using Triangular Titanium Implants vs. Non-Surgical Management: Six-Month Outcomes from a Prospective Randomized Controlled Trial. Int J Spine Surg. 2015;9:Article 6.

52. Duhon BS, Cher DJ, Wine KD, Kovalsky DA, Lockstadt H, on behalf of the SIFI Study Group. Triangular Titanium Implants for Minimally Invasive Sacroiliac Joint Fusion: A Prospective Study. Glob Spine J. 2016;6(3):257-269 [Epub 2015 Aug 11]. 53. Duhon B, Cher D, Wine K, Lockstadt H, Kovalsky D, Soo C-L. Safety and 6-month effectiveness of minimally invasive sacroiliac joint fusion: a prospective study. Med Devices Evid Res. 2013;6:219-229.
54. Capobianco R, Cher D. Safety and effectiveness of minimally invasive sacroiliac joint fusion in women with persistent post-partum posterior pelvic girdle pain: 12-month outcomes from a prospective, multi-center trial. SpringerPlus. 2015;4(1):570.

55. Sachs D. Minimally Invasive versus Open Sacroiliac Joint Fusion: A Comparison of Process Measures and Description of Technique. In: International Society for the Advancement of Spine Surgery. Vancouver, BC Canada; 2013:187.

56. Lingutla KK, Pollock R, Ahuja S. Sacroiliac joint fusion for low back pain: a systematic review and meta-analysis. Eur Spine J. March 2016:1-8. 57. Lorio M, Polly D, Ninkovic I, et al. Utilization of Minimally Invasive Surgical Approach for Sacroiliac Joint Fusion in Surgeon Population of ISASS and SMISS Membership. The Open Orthopaedics Journal January 2014;8(1):1- 6 .

58. Lorio M, Rashbaum R. ISASS Policy Statement - Minimally Invasive Sacroiliac Joint Fusion. Int $J$ Spine Surg. 2014; 8:Article 25. doi 10.14444/1025.

Published 13 July 2016. This manuscript is generously published free of charge by ISASS, the International Society for the Advancement of Spine Surgery. Copyright @ 2016 ISASS. To see more or order reprints or permissions, see http://ijssurgery.com. 